\title{
Effects of Electron Temperature Fluctuation on Turbulence Measurement by Langmuir Probe in a Magnetized Helicon Plasma
}

\author{
Yuichi KAWACHI, Shigeru INAGAKI ${ }^{1,2)}$, Makoto SASAKI ${ }^{1,3)}$, Yusuke KOSUGA ${ }^{1,2)}$, \\ Takuma YAMADA ${ }^{4)}$, Yoshihiko NAGASHIMA ${ }^{1,2)}$, Chanho MOON ${ }^{2)}$, Naohiro KASUYA ${ }^{1,2)}$ \\ and Akihide FUJISAWA ${ }^{1,2}$ \\ Interdisciplinary Graduate School of Engineering Sciences, Kyushu University, Kasuga 816-8580, Japan \\ ${ }^{1)}$ Research Center for Plasma Turbulence, Kyushu University, Kasuga 816-8580, Japan \\ ${ }^{2)}$ Research Institution for Applied Mechanics, Kyushu University, Kasuga 816-8580, Japan \\ ${ }^{3)}$ College of Industrial Technology, Nihon University, Narashino 275-8575, Japan \\ ${ }^{4)}$ Faculty of Arts and Science, Kyushu University, Fukuoka 819-0395, Japan
}

(Received 13 April 2021 / Accepted 4 May 2021)

\begin{abstract}
Effects of electron temperature fluctuation on measurement of other fluctuations by using of Langmuir probe is investigated in PANTA plasma with finite temperature gradient, where significant electron temperature fluctuations are observed. The temperature fluctuation and its effects are evaluated from spatiotemporal structures of ion saturation current and floating potential, derived by conditional sampling/averaging technique. It is found that electron density fluctuation is in phase with ion saturation current fluctuation, but plasma potential fluctuation is anti-phase with the floating potential fluctuation.
\end{abstract}

(C) 2021 The Japan Society of Plasma Science and Nuclear Fusion Research

Keywords: turbulence, transport, Langmuir probe

DOI: $10.1585 /$ pfr.16.1202081

In magnetized plasma, plasma turbulence is believed to induce large particle, momentum and energy transports. Generally, high temporal resolution is required for turbulence measurements. Langmuir probe is frequently used to measure plasma turbulence and turbulence-driven transports in fundamental plasmas and the SOL and edge region of fusion plasma $[1,2]$. The ion saturation current $\left(I_{\text {is }}\right)$ measurement and the floating potential $\left(V_{\mathrm{f}}\right)$ measurement are known to have relatively high time resolution. The $I_{\text {is }}$ and $V_{\mathrm{f}}$ are related to plasma potential $\left(V_{\mathrm{p}}\right)$ and electron density $\left(n_{\mathrm{e}}\right)$, respectively, which are important in the evaluation of turbulence fluctuation characteristics and turbulencedriven transport. The relationship between them is as follows [3];

$$
\begin{aligned}
& n_{\mathrm{e}}(t)=\exp \left(\frac{1}{2}\right) \frac{I_{\mathrm{is}}(t)}{e S} \sqrt{\frac{m_{\mathrm{i}}}{T_{\mathrm{e}}(t)}}, \\
& V_{\mathrm{p}}(t)=V_{\mathrm{f}}(t)+\alpha \frac{T_{\mathrm{e}}(t)}{e},
\end{aligned}
$$

where $t$ is time, $e$ is elementary charge, $S$ is probe surface area, $T_{\mathrm{e}}$ is electron temperature, and $m_{\mathrm{i}}$ is ion mass. $\alpha$ is given as $\alpha=\frac{1}{2}\left(1+\ln \frac{m_{\mathrm{i}}}{2 \pi m_{\mathrm{e}}}\right)$ and $m_{\mathrm{e}}$ is electron mass. Evaluations of both of electron density fluctuation $\tilde{n}_{e}$ and plasma potential fluctuation $\tilde{V}_{p}$ are affected by electron temperature fluctuation $\tilde{T}_{\mathrm{e}}$, where $\tilde{A}$ denotes fluctuation component of $A$. When the temperature gradient is smaller than the density gradient and drift waves are considered, the temperature gradient and temperature fluctuations can be neglected without changing the results [4], so they are rarely treated explicitly. However, even in the drift waves, $\tilde{T}_{\mathrm{e}}$ are excited when the temperature gradient becomes large. In recent experiments with higher injection power on PANTA, the temperature gradient is formed and finite $\tilde{T}_{\mathrm{e}}$ is observed [5]. Therefore, in this case, the $\tilde{T}_{\mathrm{e}}$ can affect to evaluations of $\tilde{n}_{\mathrm{e}}$ and $\tilde{V}_{\mathrm{p}}$. This paper reports the effects of $\tilde{T}_{\mathrm{e}}$ on the fluctuation measurements by Langmuir probe.

We have measured the temperature fluctuation of plasma in a linear device, PANTA [6]. A homogeneous axial magnetic field of $B=0.13 \mathrm{~T}$ is excited by 17 pairs of Helmholtz coils. Argon plasma with a radius of $50 \mathrm{~mm}$ is generated by RF $(7 \mathrm{MHz})$ source in $0.5 \mathrm{~Pa}$ of neutral gas pressure. The injected RF power of $6 \mathrm{~kW}$ is twice that of typical discharge on PANTA [6]. In this case, not only density gradient but also electron temperature gradient are formed [5]. Double probe measurement was carried out by using a radially movable probe array at $1625 \mathrm{~mm}$ from the RF source in shot-by-shot manners. A bias voltage of the double probe was swept at a frequency of $100 \mathrm{~Hz}$. The ratio of the gradient lengths was $\frac{\partial_{r} T_{\mathrm{e}} / T_{\mathrm{e}}}{\partial_{r} n_{\mathrm{e}} / n_{\mathrm{e}}} \sim 0.5$, where the drift wave can be destabilized and excited not only $n_{\mathrm{e}}$ but also $T_{\mathrm{e}}$. Focusing on a drift wave like fluctuation with azimuthal mode number $m=4$, we can evaluate the $\tilde{T}_{\mathrm{e}}$ and $\tilde{I}_{\mathrm{is}}$ from the double probe by using conditional sampling technique [5]. The $I_{\mathrm{is}, \text { ref }}$ signal measured by one of the probe tips of a 64-channel azimuthal probe array which is located 


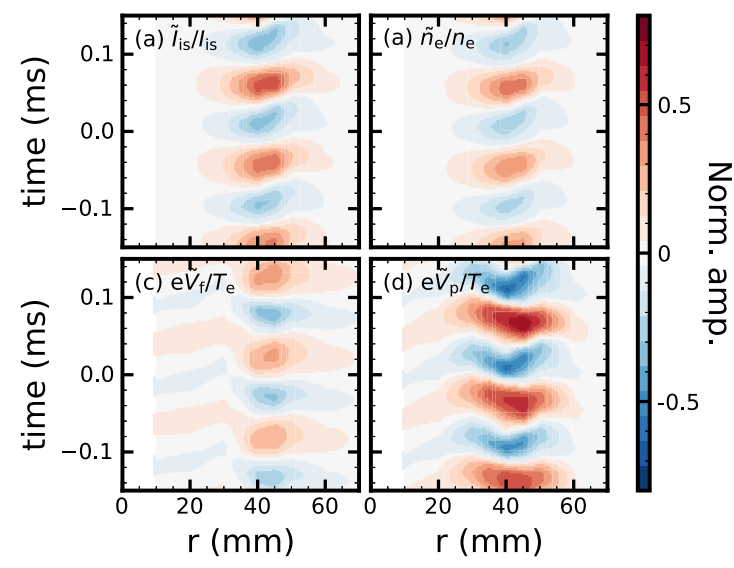

Fig. 1 The spatiotemporal evolution of the normalized fluctuations of (a) $\tilde{I}_{\text {is }} / I_{\text {is }}$, (b) $\tilde{n}_{\mathrm{e}} / n_{\mathrm{e}}$, (c) e $\tilde{V}_{\mathrm{f}} / T_{\mathrm{e}}$ and (d) e $\tilde{V}_{\mathrm{p}} / T_{\mathrm{e}}$.

$250 \mathrm{~mm}$ downstream from the double probe is utilized as a reference signal for conditional sampling [7]. The $\tilde{V}_{\mathrm{f}}$ was also measured by one of the probe tips of the radial movable probe array in the other discharges. Since we used the same reference signal $I_{\text {is,ref }}$ for the double probe measurement and the $\tilde{V}_{\mathrm{f}}$ measurement, we can evaluate the spatiotemporal structure of the $\tilde{V}_{\mathrm{f}}$ at the same time and the same position as that of the $\tilde{T}_{\mathrm{e}}$ and $\tilde{I}_{\text {is }}$ [8]. Then, the $\tilde{n}_{\mathrm{e}}$ and $\tilde{V}_{\mathrm{p}}$ were calculated from the Eqs. (1) and (2), respectively.

The spatiotemporal structures of the drift wave like fluctuation are shown in Fig. 1. All of the normalized fluctuations are peaked around $\mathrm{r}=40 \mathrm{~mm}$, where the steep density gradient is formed. Conventionally, fluctuation driven transport are estimated by using the $\tilde{I}_{\text {is }}$ and $\tilde{V}_{\mathrm{f}}$ instead of $\tilde{n}_{\mathrm{e}}$ and $\tilde{V}_{\mathrm{p}}$, respectively. However, the phase relation between $\tilde{I}_{\text {is }}$ and $\tilde{V}_{\mathrm{f}}$ can be changed from that between $\tilde{n}_{\mathrm{e}}$ and $\tilde{V}_{\mathrm{s}}$ if the $\tilde{T}_{\mathrm{e}}$ is significant. Indeed, in the experiment, contrary to the typical basic plasma, finite $\tilde{T}_{\mathrm{e}} / T_{\mathrm{e}}=0.1$ is observed and seems to affect phase and amplitude of $\tilde{V}_{\mathrm{p}}$ as shown in Figs. 1 (c) and (d).

In order to check the $\tilde{T}_{\mathrm{e}}$ effects on the phase relation, the temporal evolution of the normalized fluctuations at $r=40 \mathrm{~mm}$ is displayed in Figs. 2 (a) and (b). The normalized density fluctuation $\tilde{n}_{\mathrm{e}} / n_{\mathrm{e}}$ has the same phase and amplitude as the normalized ion saturation current fluctuation $\tilde{I}_{\text {is }} / I_{\text {is }}$. Figure 2 (c) shows the radial profile of the phase difference $\delta_{I_{\mathrm{is}}, n_{\mathrm{e}}}$ between the fundamental modes of the $\tilde{n}_{\mathrm{e}}$ and $\tilde{I}_{\mathrm{is}}$, which is evaluated by cross-spectrum analysis. The $\delta_{I_{\mathrm{s}}, n_{\mathrm{e}}}$ is almost 0 at any radial position in the region of $r \geq 20 \mathrm{~mm}$. Hence, the $\tilde{T}_{\mathrm{e}}$ effect on $\tilde{n}_{\mathrm{e}}$ evaluation is negligible, and thus the $\tilde{I}_{\text {is }}$ can be interpreted as the $\tilde{n}_{\mathrm{e}}$. While the $\tilde{T}_{\mathrm{e}}$ effect on $\tilde{V}_{\mathrm{p}}$ measurement is larger than that on $\tilde{n}_{\mathrm{e}}$ measurement. Concerning the Eq. (2), $\tilde{T}_{\mathrm{e}}$ affects to the $\tilde{V}_{\mathrm{p}}$ measurement by factor $\alpha \sim 5.2$ for argon plasma. In this case, the amplitude of $\tilde{T}_{\mathrm{e}}$ is $\sim 0.2 \mathrm{eV}$ and the effective voltage $\alpha \tilde{T}_{\mathrm{e}} / \mathrm{e}$ is $\sim 1 \mathrm{~V}$. Since the order of magnitude of $\tilde{V}_{\mathrm{f}}$ is $\sim 1 \mathrm{~V}$, the $\tilde{T}_{\mathrm{e}}$ can significantly affect to the $V_{\mathrm{p}}$ evaluation (i.e. $\tilde{V}_{\mathrm{f}} \not \tilde{V}_{\mathrm{p}}$ ). The $\tilde{V}_{\mathrm{p}}$ is anti-phase with the $\tilde{V}_{\mathrm{f}}$ as shown
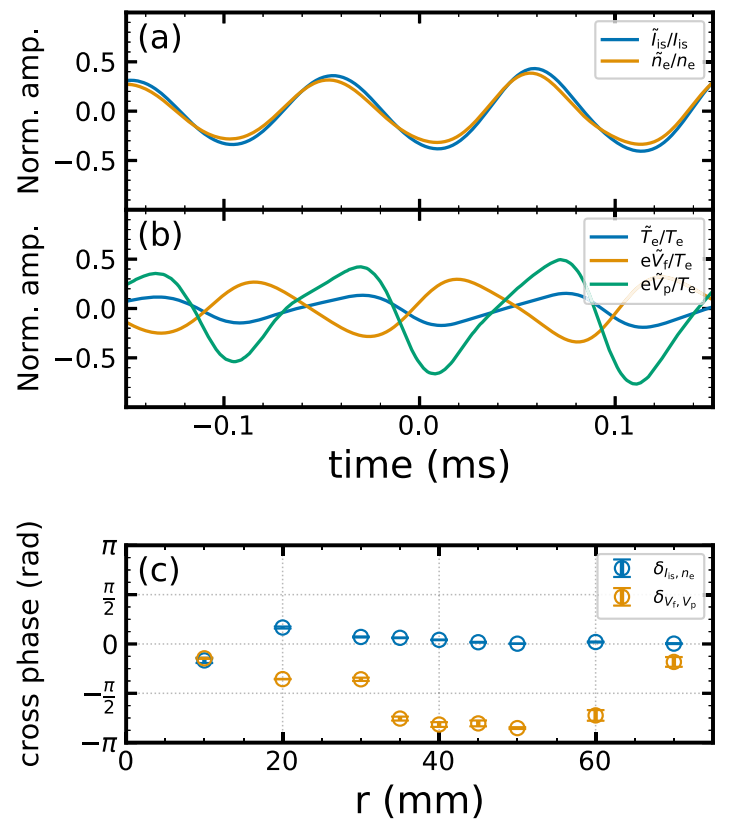

Fig. 2 Waveforms of the normalized fluctuations of (a) $\tilde{I}_{\mathrm{is}}, \tilde{n}_{\mathrm{e}}$, (b) $\tilde{T}_{\mathrm{e}}, \tilde{V}_{\mathrm{f}}$, and $V_{\mathrm{p}}$ at $r=40 \mathrm{~mm}$. (c) Radial profiles of phase differences $\delta_{n_{\mathrm{e}}, I_{\mathrm{is}}}$ and $\delta_{V_{\mathrm{p}}, V_{\mathrm{f}}}$.

in Figs. 2 (b). Radial profile of the phase difference $\delta_{V_{\mathrm{f}}, V_{\mathrm{p}}}$ between the $\tilde{V}_{\mathrm{p}}$ and $\tilde{V}_{\mathrm{f}}$ are shown in Fig. $2(\mathrm{a})$. In the region of $r=30-60 \mathrm{~mm}$, the large $\delta_{V_{\mathrm{f}}, V_{\mathrm{p}}}$ exists with up to $|\pi|$, which may cause that the direction of fluctuation driven flux evaluated by $\tilde{V}_{\mathrm{f}}$ is reversed with respect to one evaluated by $\tilde{V}_{\mathrm{p}}$. Thus, contrary to the $\tilde{n}_{\mathrm{e}}$ measurement, the $\tilde{T}_{\mathrm{e}}$ effect on the $\tilde{V}_{\mathrm{p}}$ measurement is significant . Inward particle flux evaluated by floating potential was observed in some laboratory plasmas [8-10]. As mentioned above, the presence of temperature fluctuations may result in large errors in the particle flux evaluation, so it is probably better to check for temperature fluctuation. In order to evaluate fluctuation driven transport more precisely, direct plasma potential measurement, such as emissive probe and ballpen probe is desirable and will be performed in PANTA.

In summary, we evaluated the effect of electron temperature fluctuation on measurement of density and plasma potential fluctuations. In PANTA plasma with a finite electron temperature gradient, the electron temperature fluctuation is excited, and it affects on the plasma potential fluctuation measurement and evaluation of fluctuation driven transport.

This work was supported by JSPS KAKENHI Grant Numbers JP20J12625, JP17H06089, JP17K06994, the collaboration programs of RIAM Kyushu University and of the National Institute for Fusion Science (NIFS17KOCH002, NIFS18KNWP007), and JSPS Coreto-Core Program ('PLADyS').

[1] M.J. Burin et al., Phys. Plasmas 12, 052320 (2005).

[2] O. Grulke et al., Phys. Plasmas 13, 012306 (2006). 
[3] H.M. Mott-Smith and I. Langmuir, Phys. Rev. 28, 72 (1926).

[4] N.A. Krall and M.N. Rosenbluth, Phys. Fluids 8, 1488 (1965).

[5] Y. Kawachi et al., Plasma Phys. Control. Fusion 62, 0555011 (2020).
[6] S. Inagaki et al., Sci. Rep. 6, 22189 (2016).

[7] T. Yamada et al., Nat. Phys. 4, 721 (2008).

[8] Y. Kawachi et al., Plasma Fusion Res. 14, 1402090 (2019).

[9] A. Fasoli et al., Phys. Plasmas 13, 055902 (2006).

[10] S.C. Thakur et al., Plasma Sources Sci. Technol. 23, 044006 (2014). 